\title{
Revisão rápida de esfregaços cervicais como método de garantia interna de qualidade
}

\author{
Rapid screening of cervical smears as a method of internal quality assurance
}

unitermos

Câncer de colo uterino

Teste de Papanicolaou

Revisão rápida

Garantia de qualidade

Controle de qualidade

\section{resumo}

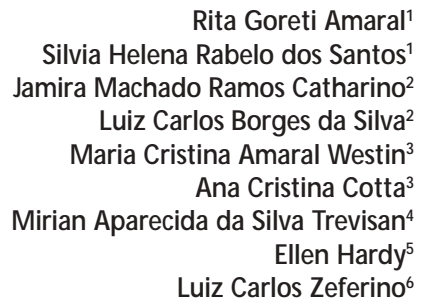

Introdução: Uma das críticas mais freqüentes ao exame citopatológico é a alta taxa de falsos

negativos. O método de garantia de qualidade mais utilizado é a revisão de $10 \%$ dos

esfregaços negativos. Segundo diversos autores, o método de revisão rápida de $100 \%$ é

eficiente para detectar resultados falsos negativos. Objetivo: Comparar o desempenho dos

métodos de revisão rápida de $100 \%$ e revisão de $10 \%$ dos esfregaços negativos como método

de garantia interna de qualidade. Métodos: Foram submetidos à revisão rápida, realizada por

um citotécnico sênior, 2.750 esfregaços com resultados negativos da rotina, seguida pela

revisão de $10 \%$ por outro citotécnico sênior. Após estas revisões, todos os esfregaços foram

analisados por dois citopatologistas, separadamente. Quando seus resultados não eram

concordantes, os esfregaços eram analisados por um terceiro citopatologista e, através de uma

reunião de consenso, era definido o diagnóstico final (padrão-ouro). Resultados: A revisão

rápida identificou 98 esfregaços suspeitos, dos quais 62 foram confirmados pelo padrão-ouro,

sendo 45 Ascus, 11 LSIL e seis HSIL (sensibilidade 73,8\%). A revisão de 10\% identificou nove

esfregaços positivos, dos quais seis foram confirmados pelo padrão-ouro, sendo três Ascus, dois

LSIL e um HSIL (sensibilidade 50\%). Dos 2.489 esfregaços não-incluídos na revisão de 10\%, o

padrão-ouro identificou 57 Ascus, dez LSIL e cinco casos HSIL. Conclusão: A revisão rápida de

$100 \%$ é uma alternativa eficiente para reduzir as taxas de falsos negativos dos exames

citopatológicos. Permite ainda o monitoramento do desempenho individual da equipe.

\section{abstract}

Introduction: One of the most frequently cited disadvantages of cervical cancer screening is its high

false negative rate. The most widely used quality assurance method is $10 \%$ random re-screening.

Recent studies have shown that $100 \%$ rapid re-screening is an efficient method for reduction of the false negative rate. Objective: The aim of this study was to compare the performance of $100 \%$ rapid re-screening with that of $10 \%$ random re-screening as an internal quality assurance method.

M ethods: 2,750 first screening negative smears were submitted to $100 \%$ rapid re-screening by a senior cytotechnologist followed by $10 \%$ random re-screening by another senior cytotechnologist.

After re-screening, all smears were analyzed by two independent cytopathologists. Whenever results

were divergent the smears were analyzed by a third cytopathologist and the panel established a

consensus gold standard diagnosis. Results: 98 suspect smears were selected by rapid re-screening,

62 of which were confirmed as abnormal by the gold standard: 45 Ascus, 11 LSIL and six HSIL

(sensitivity $73.8 \%$ ). Of the nine abnormal smears detected by the $10 \%$ random re-screening, six were confirmed by the gold standard, three Ascus, two LSIL and one HSIL (sensitivity 50\%). The gold

standard detected 57 Ascus, ten LSIL and five HSIL cases among the 2,489 smears that were not

submitted to $10 \%$ re-screening. Conclusions: $100 \%$ rapid re-screening is an efficient alternative for

reduction of cervical screening false negative rates. It also allowed to monitor the individual performance of members of the team. 


\section{Introdução}

Uma das críticas mais freqüentes ao exame citopatológico é a alta taxa de falsos negativos, que varia de $6 \%$ a $68 \%$, chegando a pôr em dúvida sua validade na prevenção e na detecção precoce de câncer de colo uterino $(10,11,19,22)$. Segundo Gay (9), as principais causas que levam a resultados falsos negativos foram atribuídas a erros na colheita de material (62\%), no escrutínio do esfregaço (16\%) e na interpretação dos achados citológicos (22\%).

Têm sido avaliados vários métodos, com diferentes custos e eficiência, para garantia de qualidade do diagnóstico citopatológico. O mais utilizado é a revisão de 10\% dos esfregaços negativos $(1,13,14,17)$. Entretanto, o fato de revisar apenas $10 \%$ dos esfregaços parece não ter sido eficiente para reduzir as taxas de falsos negativos (4, $18,23)$. A rigor, mesmo que se detecte alguma discordância diagnóstica na amostra de $10 \%$, não se revisam os $90 \%$ restantes, e eventuais deficiências ou diferenças dos profissionais podem demorar a ser detectadas. Também tem sido utilizado sistema automatizado para o diagnóstico citopatológico, todavia de forma restrita, devido ao alto custo dos equipamentos e de sua operacionalização (12, $16,21,24)$.

Alguns estudos têm avaliado a revisão rápida de 100\% dos esfregaços negativos para garantia de qualidade, porém este método exige grande experiência e agilidade do revisor $(3,5-8)$. Recentemente, alguns estudos têm mostrado que a revisão rápida de $100 \%$ pode reduzir a taxa de falsos negativos e substituir o método de revisão de $10 \%(2,8,15,20)$, mas esta proposta precisa ser testada em diferentes serviços para verificar se há concordância nos resultados. Portanto o objetivo deste estudo foi comparar, em um laboratório de grande porte, o desempenho dos métodos de revisão rápida de $100 \%$ e revisão de $10 \%$ dos esfregaços negativos da rotina, para identificar aquele que oferece maior garantia interna de qualidade.

\section{Material e métodos}

Este estudo foi realizado no Laboratório de Citopatologia do Caism/Unicamp, que analisa uma média de 21 mil esfregaços por mês. Atualmente, os citopatologistas revêem os esfregaços positivos ou com diagnóstico duvidoso e os esfregaços de pacientes com história clínica relevante. Os citotécnicos seniores revisam $10 \%$ dos esfregaços negativos.
Entre fevereiro e julho de 2001, foram selecionados 2.750 esfregaços citológicos com resultado negativo na análise da rotina. Diariamente foi sorteado um citotécnico e selecionados os primeiros 40 esfregaços negativos analisados no dia anterior. Estes esfregaços foram submetidos à revisão rápida por um dos citotécnicos seniores. De cada grupo de 40 esfregaços foram sorteados quatro, que foram submetidos à revisão de $10 \%$ por outro citotécnico sênior. Após estas revisões, todos os esfregaços foram analisados separadamente por dois citopatologistas, e os resultados foram anotados separadamente. Nos casos em que os resultados foram discordantes, os esfregaços foram analisados por um terceiro citopatologista. $\mathrm{O}$ diagnóstico final (padrão-ouro) foi definido durante uma reunião de consenso. Os resultados das revisões dos citotécnicos seniores e dos citopatologistas foram mantidos em sigilo, à exceção daqueles discutidos durante a reunião de consenso.

Participaram do estudo quatro citotécnicos seniores, com pelo menos 15 anos de experiência, que se alternaram semanalmente nas funções de revisores da revisão rápida $100 \%$ e revisão de $10 \%$. Os citotécnicos foram treinados durante dois meses para realizarem a revisão rápida, visto que o laboratório não utilizava esta metodologia como garantia interna de qualidade. O tempo adotado para revisão rápida de cada esfregaço foi de um minuto cronometrado, devendo ser analisados pelo menos quarenta campos através da objetiva de 10X. A análise de consistência dos resultados e o manuseio dos formulários foram realizados por um pesquisador que não participou das revisões.

\section{Resultados}

A revisão rápida de 100\% identificou 98 esfregaços suspeitos, sendo que dois terços foram confirmados pelo padrão-ouro. Dos 261 esfregaços encaminhados para revisão de $10 \%$, nove foram considerados positivos, sendo que dois terços foram confirmados pelo padrão-ouro (Tabela 1).

Dos 62 esfregaços positivos identificados pela revisão rápida, 45 foram classificados como células escamosas atípicas de significado indeterminado (Ascus), 11 como lesão intra-epitelial escamosa de baixo grau (LSIL) e seis como lesão intra-epitelial escamosa de alto grau (HSIL). Todos os esfregaços de HSIL classificados pelo padrão-ouro foram detectados pela revisão rápida. Nenhum caso de carcinoma invasivo foi detectado. A sensibilidade global 


\section{Esfregaços alterados detectados pela revisão rápida de $100 \%$ e pela revisão de $10 \%$}

Tabela 1 e confirmados pelo padräo-ouro

\begin{tabular}{lcc}
\hline Categoria & $n$ & $\%$ \\
\hline Total de casos do estudo & 2.750 & 100 \\
\hline Esfregaços para revisão rápida & 2.750 & 100 \\
Revisão rápida com esfregaços suspeitos & 98 & 3,6 \\
Confirmados pelo padrão-ouro & 62 & - \\
Não-confirmados pelo padrão-ouro & 36 & - \\
Revisão rápida com esfregaços negativos & 2.652 & 96,4 \\
Esfregaços para revisão 10\% & 261 & $9,5^{*}$ \\
Revisão 10\% com esfregaços positivos & 9 & 0,3 \\
Confirmados pelo padrão-ouro & 6 & - \\
Não-confirmados pelo padrão-ouro & 3 & - \\
Revisão 10\% com esfregaços negativos & 252 & 9,2 \\
Não-analisados pela revisão 10\% & 2.391 & 90,5 \\
\hline
\end{tabular}

*0 número relativo de casos analisados na revisão de $10 \%$ foi de $9,5 \%$ devido ao fato de alguns casos terem sido excluídos da análise por serem classificados como insatisfatórios pelo padrão-ouro.

da revisão rápida foi de 73,8\%. Para os diagnósticos de Ascus, LSIL e HSIL, a sensibilidade foi de $68 \%$, $92 \%$ e $100 \%$, respectivamente. Dos 98 esfregaços suspeitos, detectados pela revisão rápida, $37 \%$ foram falsos alarmes, pois o padrão-ouro não confirmou alterações citológicas. Dos 84 esfregaços positivos detectados pelo padrão-ouro, 21 Ascus e um LSIL foram considerados negativos pela revisão rápida (Tabela 2).

Dos seis esfregaços positivos detectados pela revisão de $10 \%$ e confirmados pelo padrão-ouro, três foram classificados como Ascus, dois como LSIL e apenas um como HSIL. A sensibilidade da revisão de $10 \%$ foi de $50 \%$, devido às discordâncias nos diagnósticos de Ascus, mesmo havendo concordância com o padrão-ouro nos diagnósticos de LSIL e HSIL. Dos 2.489 esfregaços não-analisados pelo método de revisão de 10\%, o padrão-ouro identificou 57 Ascus, dez LSIL e cinco HSIL (Tabela 3).

\section{Discussão}

Os resultados deste estudo mostram que o desempenho do método de revisão rápida de $100 \%$ é eficiente como garantia interna de qualidade, que visa a detectar as alterações citológicas não-identificadas no primeiro escrutínio. Por outro lado, a revisão de $10 \%$ tem uma forte limitação por não submeter os outros $90 \%$ dos esfregaços negativos à revisão para garantia interna de qualidade.
De 84 esfregaços com diagnósticos falsos negativos identificados pelo padrão-ouro, 62 foram detectados pela revisão rápida, que incluiu todos os esfregaços com diagnóstico de HSIL. Os casos não-detectados foram 21 Ascus e um LSIL, ou seja, diagnósticos considerados limítrofes.

Um estudo de revisão rápida de 100\% detectou 99 esfregaços suspeitos, dentre os quais 58 foram confirmados pela revisão detalhada e classificados como 43 de Ascus ou Agus, 14 de LSIL e um caso de carcinoma escamoso invasivo (5). Em outro estudo, de 2.925 esfregaços, a revisão rápida de $100 \%$, utilizando o tempo de um minuto, detectou um adicional de nove Ascus, dez LSIL e dois HSIL (8). Outros autores encontraram, através da revisão rápida de $100 \%$, um maior número de falsos negativos (120 alterações borderline, 27 LSIL e 14 HSIL) quando em comparação com a revisão aleatória de 10\% (21 alterações borderline, quatro LSIL e um HSIL) e com a revisão de casos clinicamente indicados (19 alterações borderline e cinco LSIL). Estes autores não encontraram diferença significativa entre as taxas de falsos negativos da revisão de $10 \%$ e as da revisão dos casos clinicamente indicados. Sugerem que os erros do escrutínio primário não são mais freqüentes em pacientes de alto risco, ainda que a prevalência de lesões seja maior nestes (4). Nenhum dos estudos citados realizou revisão dos esfregaços considerados negativos pela revisão rápida, de tal forma que não dispõem de informações sobre os falsos negativos da revisão rápida. 


\begin{tabular}{|c|c|c|c|c|c|}
\hline \multirow{3}{*}{$\begin{array}{l}\text { Tabela } 2 \\
\text { Revisão rápida }\end{array}$} & \multicolumn{5}{|c|}{$\begin{array}{l}\text { Achados suspeitos pela revisão } \\
\text { rápida de } 100 \% \text { e } 0 \text { diagnóstico } \\
\text { estabelecido pelo padrão-0uro }\end{array}$} \\
\hline & & Padr & 30-ouro & & Total \\
\hline & Ascus & LSIL & HSIL & Negativo & \\
\hline $\begin{array}{l}\text { Esfregaços } \\
\text { suspeitos }\end{array}$ & 45 & 11 & 6 & 36 & 98 \\
\hline Negativo & 21 & 1 & 0 & 2.630 & 2.652 \\
\hline Total & 66 & 12 & 6 & 2.666 & 2.750 \\
\hline
\end{tabular}

Para calcular a sensibilidade e 0 valor preditivo positivo considerou-se achado positivo do padrão-ouro a soma dos diagnósticos de Ascus, LSIL e HSIL; sensibilidade: 73,81\% - IC 95\% (62,88\% a 82,53\%); especificidade: $98,65 \%$ - IC $95 \%$ (98,11\% a $99,04 \%)$; valor preditivo positivo: $63,27 \%$; valor preditivo negativo: $99,17 \%$.

Tabela 3

\begin{tabular}{|c|c|c|c|c|c|}
\hline \multirow[t]{2}{*}{ Revisão 10\% } & \multicolumn{4}{|c|}{ Padrão-ouro } & \multirow[t]{2}{*}{ Total } \\
\hline & Ascus & LSIL & HSIL & Negativo & \\
\hline $\begin{array}{c}\text { Esfregaços } \\
\text { Suspeitos }\end{array}$ & 3 & 2 & 1 & 3 & 9 \\
\hline Negativo & 6 & 0 & 0 & 246 & 252 \\
\hline Subtotal & 9 & 2 & 1 & 249 & 261 \\
\hline $\begin{array}{l}\text { Não- } \\
\text { realizada* }\end{array}$ & 57 & 10 & 5 & 2.417 & 2.489 \\
\hline Total & 66 & 12 & 6 & 2.666 & 2.750 \\
\hline
\end{tabular}

Para calcular a sensibilidade e 0 valor preditivo positivo considerou-se achado positivo do padrão-ouro a soma dos diagnósticos de Ascus, LSIL e HSIL; sensibilidade: 50\% - IC 95\% (22,9\% a 77,7\%); especificidade: $98,8 \%$ - IC $95 \%$ (96,23\% a $99,6 \%)$; valor preditivo positivo: $66,7 \%$; valor preditivo negativo: $97,2 \%$. *Esfregaços que não foram incluídos na revisão de $10 \%$.

A técnica e o tempo da revisão, o desempenho dos indivíduos e o limite de trabalho diário foram avaliados em um estudo comparativo realizado em vários laboratórios. Os autores concluíram que a revisão rápida de 100\% é um bom método de garantia interna de qualidade. En- tretanto sugeriram que os laboratórios devem padronizar o tempo (60 segundos por lâmina), cada revisor não deve ultrapassar 50 lâminas por sessão de leitura rápida e os revisores devem ser cuidadosamente treinados (6).

A preocupação inicial neste estudo foram o treinamento, a escolha da técnica e a definição do tempo a serem utilizados. Somente se iniciou a pesquisa após um período de dois meses de treinamento. A revisão rápida foi a primeira atividade diária dos revisores com limite de 40 esfregaços.

É esperado que a revisão rápida detecte lesões menos graves, pois seriam aquelas com alterações celulares qualitativamente menos evidentes no primeiro escrutínio, o que foi observado neste estudo. Assim, $76 \%$ dos esfregaços identificados pela revisão rápida e $79 \%$ dos esfregaços detectados pelo padrão-ouro tiveram o diagnóstico de Ascus. Os seis esfregaços de HSIL detectados nas revisões seriam classificados como neoplasia intra-epitelial cervical grau 2 (NIC 2), segundo Ralph Richart.

Portanto a revisão rápida de $100 \%$ é uma alternativa eficiente para reduzir a taxa de falsos negativos dos exames citopatológicos, que é uma das principais críticas que têm sido feitas a este método de diagnóstico. A rigor, permite que se monitore o desempenho individual, detectando deficiências, diferenças ou dificuldades dos profissionais com relação à interpretação diagnóstica, que é muito importante em um laboratório de grande porte. É possível que os citotécnicos responsáveis pelo escrutínio fiquem mais atentos e concentrados pelo fato de que todas as lâminas serão revisadas e mais interessados em participar de programas de educação continuada.

\section{Agradecimentos}

Aos citotécnicos seniores Luiz Fassina Neto, Luiz Carlos Borges da Silva e Eliana Borin Lopes Montemor, ao médico citopatologista dr. Douglas Munhoz e ao estatístico Edson Zangiacomi Martinez pela grande contribuição na realização deste trabalho.

\section{Referências}

1.A lves,V.A.F.et al. Programa de controle de qualidade em citologia ginecoló gica do Instituto A do lfo Lutz: estratégias e análise crítica dos resultados de sua implantação piloto. Rev. Ass. M éd. Brasil, 37: 36-42, 1991.

2.A rbyn, M.\& Schenck, U. D etection of false negative Pap smears by rapid reviewing. Acta Cytol., 44: 949-57, 2000.
3. Baker, A. \& Melcher, D. Rapid cervical cytology screening. Cytopathol., 2: 299-301, 1991.

4. Baker, A.; Melcher, D. \& Smith, R. Role of re-screening of cervical smears in internal quality control.J.Clin. Pathol.48:1002-4,1995.

5. D iehl, A.R.S. \& Prolla, J.C. Rapid rescreening of cervical smears for internal quality control. Acta Cytol., 42: 949-53, 1998. 
6. Dudding, $N$. et al. Rapid screening: a comparative study. Cytopathol., 12: 235-48, 2001.

7.Faraker, C.A.\& Boxer,M.E. Rapid review (partial rescreening) of cervical cytology. Four year experience and quality assurance implications. J. Clin. Pathol., 49: 587-91, 1996.

8. Farrell, D J. et al. Rapid screening of cervical smears as a method of internal quality control:for how long should we rescreen? Acta Cytol., 41:251-60, 1997.

9. Gay, J.D .; D onaldson, L.D. \& G oellner, J.R. False-negative results in cervical cytology studies. Acta Cytol., 29:1043-6, 1985.

10. G onçalves, W .J.; G irão, M .J.B.C . \& Bortoletto, C.C.R. Rastreamento e diagnóstico precoce das neoplasias malignas genitais: perspectivas do arsenal propedêutico.J. Bras. M éd., 69: 14-23, 1995.

11. Guimarães, E.M. \& Silva,A.M . Erros em cito patologia ginecológica: por que ocorrem? J. Bras. Ginec., 105: 397-404, 1995.

12. Halford,J.A .;W hight, R.G.\& Ditchmen, E.J. Q uality assurance in cervical cytology screening:comparison of rapid rescreening and the Papnet testing system. Acta Cythol.,4 1:79-81, 1997.

13. H utchinson, M.L.A ssessing the costs and benefits of alternative rescreening strategies. Acta Cythol., 40: 4-8, 1996.

14. Kraemer, B.B. Q uality assurance activities of the College of A merican Pathologists. Acta Cytol., 33: 343-8, 1989.

15. Lemay, C.\& Meisels, A. 100\% rapid (partial) rescreening for quality assurance. Acta Cytol., 43: 86-9, 1999.
16. Linder,J.\& Zahniser,D.ThinPrep Papanicolaou testing to reduce false-negative cervical cytolo gy. Arc. Pathol. Lab. M ed., 122: 139-44, 1998.

17. Melamed, M.R.\& Flehinger, B.J. Reevaluation of quality assurance in the cytology laboratory. Acta Cytol., 36:461-5, 1992.

18. Melamed, M.R. Rescreening for quality control in cytology. Acta Cytol., 40: 12-3, 1996.

19. Mitchell, H.; Medley, G.\& Drake, M. Q uality control measures for cervical cytology laboratories. Acta Cytol., 32: 288-92, 1988.

20. Renshaw, A.A. et al. False negative rate of cervical cytologic smear screening as determined by rapid rescreening. Acta Cytol., 43: 344-50, 1998.

21. Rosenthal, D.L.; Costa, A.D. \& Peters, R.K. Computer-assisted rescreening of clinically important false negative cervical smears using the Papnet testing system. Acta Cytol., 40: 120-6, 1996.

22. Shirata, N.K.; Pereira, S.M.M \& \& Cavaliere, M.J.C elularidade dos esfregaços cervicovaginais: importância em programas de garantia de qualidade. J. Bras. Ginec., 108: 63-6, 1998.

23. Tabbara, S.O. \& Sidawy, M.K. Evaluation of $10 \%$ rescreen of negative gynecologic smears as a quality assurance measure. Diagn. Cytopathol, 14: 84-6, 1996.

24. Vooijs, G.P. et al. Cytosafe-plus: a workstation for screening, supervision, reviewing, quality assurance and education in cyto pathology. Acta. Cytol., 40: 90-6, 1996. 